\title{
Indirect Enzyme-Linked Immunosorbent Assay for Detection of Brucella melitensis-Specific Antibodies in Goat Milk
}

\author{
N. D. Funk, ${ }^{1}$ L. B. Tabatabai, ${ }^{2}$ P. H. Elzer, ${ }^{3}$ S. D. Hagius, ${ }^{3}$ B. M. Martin, ${ }^{4 *}$ and L. J. Hoffman ${ }^{5}$ \\ Department of Veterinary Microbiology and Preventive Medicine ${ }^{1}$ and Veterinary Diagnostic Laboratory, ${ }^{5}$ College of Veterinary \\ Medicine, Iowa State University, and Agricultural Research Service, National Animal Disease Center, ${ }^{2}$ and National \\ Veterinary Services Laboratories, ${ }^{4}$ U.S. Department of Agriculture, Ames, Iowa, and Department of \\ Veterinary Science, Louisiana State University AgCenter, Baton Rouge, Louisiana ${ }^{3}$
}

Received 19 March 2004/Returned for modification 10 June 2004/Accepted 7 August 2004

\begin{abstract}
Brucella melitensis is the cause of brucellosis in sheep and goats, which often results in abortion. Few cases of $B$. melitensis infection in goats have occurred in the United States over the last 25 years. However, vigilance must be maintained, as it is for the bovine milk industry, to ensure that brucellosis is not introduced into the U.S. goat population. The objective of this study was to develop a sensitive and specific indirect enzyme-linked immunosorbent assay (iELISA) for the detection of $\boldsymbol{B}$. melitensis-specific antibodies in goat milk. Brucella salt-extractable protein extract was employed as an antigen, and a horseradish peroxidase-labeled polyclonal anti-goat antibody was used as an anti-species conjugate. Thirteen of $13(100 \%)$ individual infected goat milk samples tested positive and 134 of $134(100 \%)$ uninfected bulk milk samples tested negative by the developed iELISA. Three positive milk samples with high, medium, and low absorbance values were used to simulate one positive animal in an otherwise negative herd. By this estimation, one high-titer animal could be detected in a herd of $>1,600$ animals. Detection estimates for medium- and low-titer animals were one positive animal per herd of $<200$ and 50 animals, respectively. Based on this estimation, it is recommended that herds be sampled in groups of $\mathbf{5 0}$ animals or less for bulk milk testing. The iELISA developed for this study was found to be sensitive and specific and shows potential for use as a bulk milk test for the detection of $\boldsymbol{B}$. melitensis-specific antibodies in goat milk.
\end{abstract}

Brucella species have impacted human and animal health for thousands of years $(4,18)$. Brucellae cause disease in goats, cattle, sheep, pigs, dogs, marine mammals, and several wild animals. The focus of this work was to develop a sensitive and specific diagnostic test for the detection of anti-brucella antibodies in goat milk. Goats are the natural hosts for Brucella melitensis. Although the United States has reported the eradication of B. melitensis in animals since 1972 (5), sporadic outbreaks have occurred in relation to infected imported goats $(10,23)$. For the health of American goat milk consumers, vigilance in brucella detection must continue for the goat milk industry just as it has for the bovine milk industry.

Brucella detection assays for goats are nearly the same as those for cattle because of the considerable genetic similarity between $B$. melitensis and $B$. abortus, and they have been extensively reviewed elsewhere (14). All of these methods have primarily focused on the use of serum as a diagnostic specimen. To date, little has been done to develop and validate diagnostic tests to detect $B$. melitensis infections by using goat milk. The detection of $B$. abortus in cow milk has been successful for years by use of the milk ring test (19). Because of a difference between the physiologic properties of goat and cow milk, the milk ring test does not perform well with goat samples (13).

The objective of this study was to develop an indirect enzyme-linked immunosorbent assay (iELISA) for the detection

\footnotetext{
* Corresponding author. Mailing address: National Veterinary Services Laboratories, U.S. Department of Agriculture, 1800 Dayton Ave., Ames, IA 50010. Phone: (515) 663-7731. Fax: (515) 663-7397. E-mail: Barbara.M.Martin@aphis.usda.gov.
}

of B. melitensis-specific antibodies in goat milk. Brucella saltextractable protein extract (BCSP) has been used as an antigen for the detection of B. abortus in cattle (21) and is used here as an antigen for iELISA.

\section{MATERIALS AND METHODS}

Antigen preparation. A whole-cell sonicate (WCS) was prepared from heatkilled B. melitensis strain $16 \mathrm{M}$ (National Veterinary Services Laboratories, Ames, Iowa). Cells were sonicated at $30 \mathrm{~Hz}$ for $15 \mathrm{~min}$ with a Sonifier 250 (Branson Ultrasonics Corp., Danbury, Conn.).

Brucella salt-extractable proteins were prepared as described previously (20). Methanol-killed B. abortus strain 1119-3 cells (National Veterinary Services Laboratories) were combined with $1 \mathrm{M} \mathrm{NaCl}-0.1 \mathrm{M}$ sodium citrate $(0.2 \mathrm{~g}$ per ml) and stirred overnight at $5^{\circ} \mathrm{C}$. The suspension was centrifuged at $10,000 \times g$ at $5^{\circ} \mathrm{C}$ for $20 \mathrm{~min}$. This process was repeated, and the supernatants were then combined

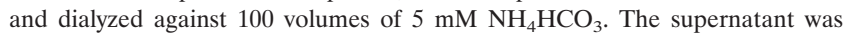
again centrifuged at $10,000 \times g$ for $30 \mathrm{~min}$ at $5^{\circ} \mathrm{C}$ and then was precipitated with solid $\left(\mathrm{NH}_{4}\right)_{2} \mathrm{SO}_{4}$ at $70 \%$ saturation for $16 \mathrm{~h}$ at $5^{\circ} \mathrm{C}$. The precipitate was centrifuged at $15,000 \times g$, reconstituted in a minimal volume of $5 \mathrm{mM} \mathrm{NH}_{4} \mathrm{HCO}_{3}$, and dialyzed against 100 volumes of $5 \mathrm{mM} \mathrm{NH}_{4} \mathrm{HCO}_{3}$. The resulting solution was freeze-dried, adjusted to a concentration of $1.0 \mathrm{mg}$ of protein $/ \mathrm{ml}$, and stored at $-70^{\circ} \mathrm{C}$. Protein, carbohydrate, and 2-keto-3-deoxyoctulosonic acid (KDO) were measured as described previously $(7,12,21,22)$.

Animal inoculation. Sixteen female goats were inoculated with a $50-\mu l$ volume containing $10^{7} \mathrm{CFU}$ of $B$. melitensis strain $16 \mathrm{M}$ into each conjunctival sac (100 $\mu l$ total). This work was performed at the Louisiana State University AgCenter in a state- and U.S. Department of Agriculture-approved large-animal isolation unit. The mean time to necropsy was 38 days (range, 15 to 50 days). B. melitensis strain $16 \mathrm{M}$ was isolated from tissues and milk of all infected goats $(1,8)$. Serum samples from all goats were positive by the card test (1).

Milk samples. Milk samples from experimentally inoculated animals which were positive by both the card test and cell culture were obtained at necropsy. Sixteen positive milk samples were received from the Louisiana State University AgCenter. Three mucoid samples were not used because their consistency made precise volume measurements impossible. One-half of a milliliter of each of the remaining 13 positive samples was combined for use as a pooled positive control for tests of assay specificity with negative milk samples. 
TABLE 1. Ratios of carbohydrate and KDO from WCS and BCSP to total protein concentrations

\begin{tabular}{lcc}
\hline Antigen & $\begin{array}{c}\text { Carbohydrate/protein ratio } \\
(\mathrm{mg} / \mathrm{mg})\end{array}$ & $\begin{array}{c}\mathrm{KDO} / \mathrm{protein} \text { ratio } \\
(\mu \mathrm{g} / \mathrm{mg})\end{array}$ \\
\hline BCSP & 0.31 & 7.86 \\
WCS & 0.014 & 4.83 \\
\hline
\end{tabular}

Negative milk samples used for specificity determinations came from bulk milk samples from 134 goat herds in the United States; samples were obtained from Wisconsin (69), California (30), Vermont (21), Michigan (7), and New York (7). Herd sizes ranged from 20 to 1,200 animals (median $=95$ ). These samples were assumed to be negative for brucellae, as $B$. melitensis has been eradicated from the United States since 1972 (5) and there was no history of chronic abortions in any herd. A pooled milk sample from three individual healthy goats was used as a negative control throughout the experiment. The cream was separated and removed from all milk samples by centrifugation at $2,000 \times g$ for $20 \mathrm{~min}$ before testing.

iELISA for antibodies in milk. The iELISA procedure was performed as previously described (21). Briefly, 96-well plates (Nalge Nunc International, Rochester, N.Y.) were coated with $100 \mu$ l of $0.1-\mu \mathrm{g} / \mathrm{ml}$ BCSP or WCS suspended in $0.05 \mathrm{M} \mathrm{Na}_{2} \mathrm{CO}_{3}$ and then incubated overnight at $5^{\circ} \mathrm{C}$. The plates were washed five times with $0.1 \mathrm{M}$ phosphate-buffered saline plus $0.05 \%$ Tween 80 (PBST) (Difco Laboratories, Detroit, Mich.) for $5 \mathrm{~min}$ each time. One hundred microliters of a 1/100 dilution of milk in PBST was plated and incubated for $2 \mathrm{~h}$ at room temperature. The plates were again washed with PBST as described earlier. One hundred microliters of horseradish peroxidase-conjugated rabbit polyclonal anti-goat immunoglobulin $\mathrm{G}$ (heavy plus light chains) (Bethyl Laboratories, Inc., Montgomery, Tex.) diluted 1/2,000 was added to each well of the plate. The plates were again incubated for $2 \mathrm{~h}$ at room temperature or overnight at $5^{\circ} \mathrm{C}$. The plates were then washed with PBST and a single wash of distilled water before

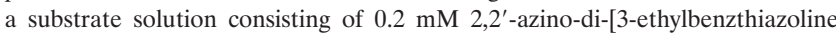
sulfonate] (6) (ABTS; Sigma Chemical Co., St. Louis, Mo.) and $5 \mathrm{mM} \mathrm{H}_{2} \mathrm{O}_{2}$ $(0.015 \%)$ in $50 \mathrm{mM}$ citrate buffer ( $\mathrm{pH} 4.0)$ was added to produce a color change. The reaction was stopped after $10 \mathrm{~min}$ by the addition of $1 \mathrm{mM}$ sodium azide. A plate reader (Bio-Tek Instruments, Winooski, Vt.) was used to read the absorbance at $492 \mathrm{~nm}$. All samples were tested in duplicate, with average absorbance values being reported. Antigens, milk samples, and the anti-species conjugate were titrated for optimal assay performance.
Positive bulk milk simulation. To simulate the presence of one positive animal in a bulk milk sample, we chose high-, medium-, and low-titer positive milk samples and serially diluted them in $B$. melitensis-negative milk. The resulting dilutions were tested at a 1/10 dilution in PBST, and the average absorbance values from two separate trials were reported. This estimation assumed that equal volumes of milk were produced by infected and uninfected goats.

\section{RESULTS}

Chemical assays. BCSP extract and WCS were both investigated for the ability to bind brucella antibodies in infected goat milk. The results of chemical assays performed with BCSP and WCS are presented in Table 1. When expressed as proportions of the protein concentration, both the KDO content and the carbohydrate concentration were higher for the BCSP antigen than for WCS.

Comparison of BCSP and WCS antigens. There was a marked difference in the absorbance values of pooled positive milk against BCSP and those against WCS (Fig. 1). The maximal absorbance from a pooled positive milk sample with BCSP as the antigen was 0.8 absorbance units, in contrast to 0.4 absorbance units with WCS as the antigen. The titration kinetics with positive pooled milk and BCSP showed a larger change in absorbance as a function of the milk dilution than that with WCS. The titration kinetics with negative pooled milk were very similar for both BCSP and WCS. Only BCSP was used to determine the sensitivity and specificity of detection.

Sensitivity and specificity. Single 1/100 dilutions of individual positive and duplicate negative milk samples were tested by iELISA, and absorbance values were reported (Fig. 2). Table 2 lists the absorbance values of the individual positive milk samples, the times (days postinfection) of sample collection, and the numbers of CFU detected in $1 \mathrm{ml}$ of milk. The absorbance values for the positive milk samples ranged from 0.35 to 0.94 , whereas the absorbance values for the negative milk samples

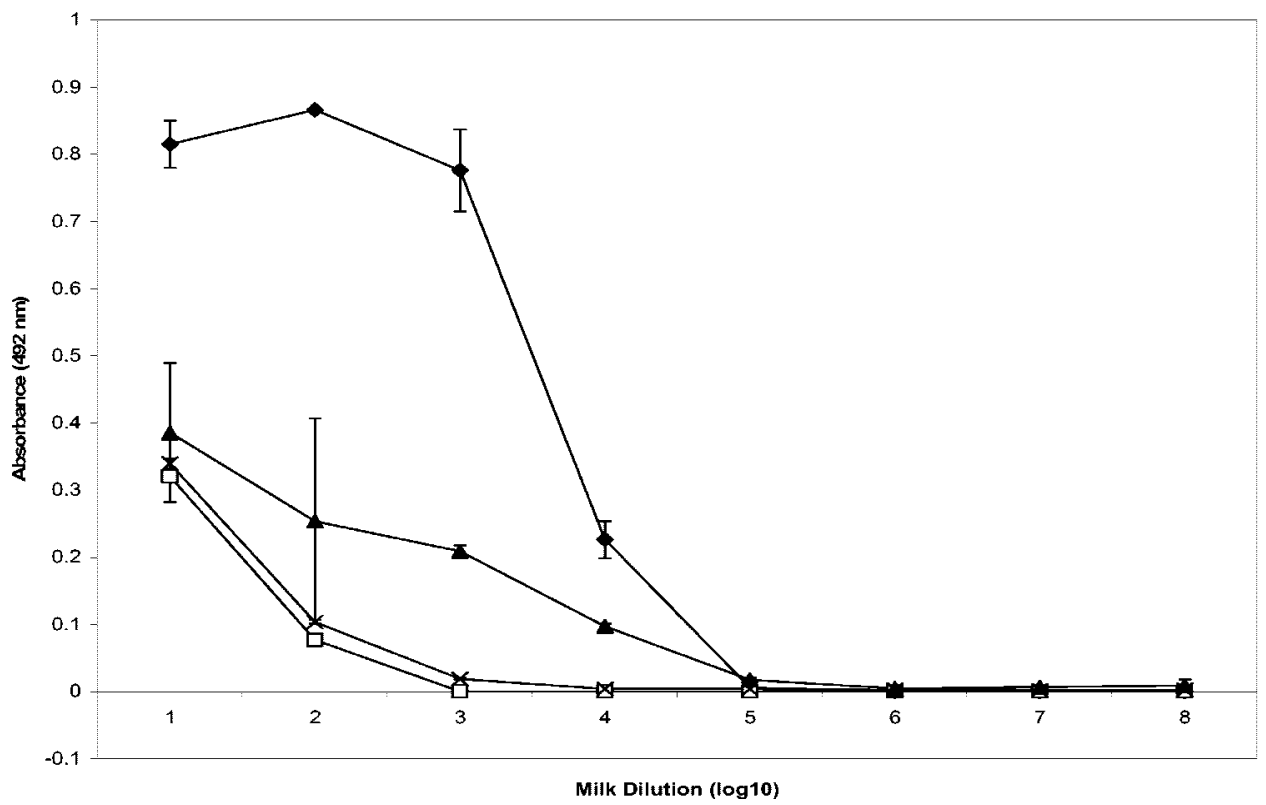

FIG. 1. Comparison of BCSP and WCS as antigens. Anti-brucella antibody responses were compared by iELISAs with pooled positive milk ( $\bullet$, BCSP; $\boldsymbol{\Delta}$, WCS) and negative control milk samples ( $\square$, BCSP; $\times$, WCS). Data points represent the average absorbance values of duplicate wells. Bars indicate the ranges of absorbance values. 


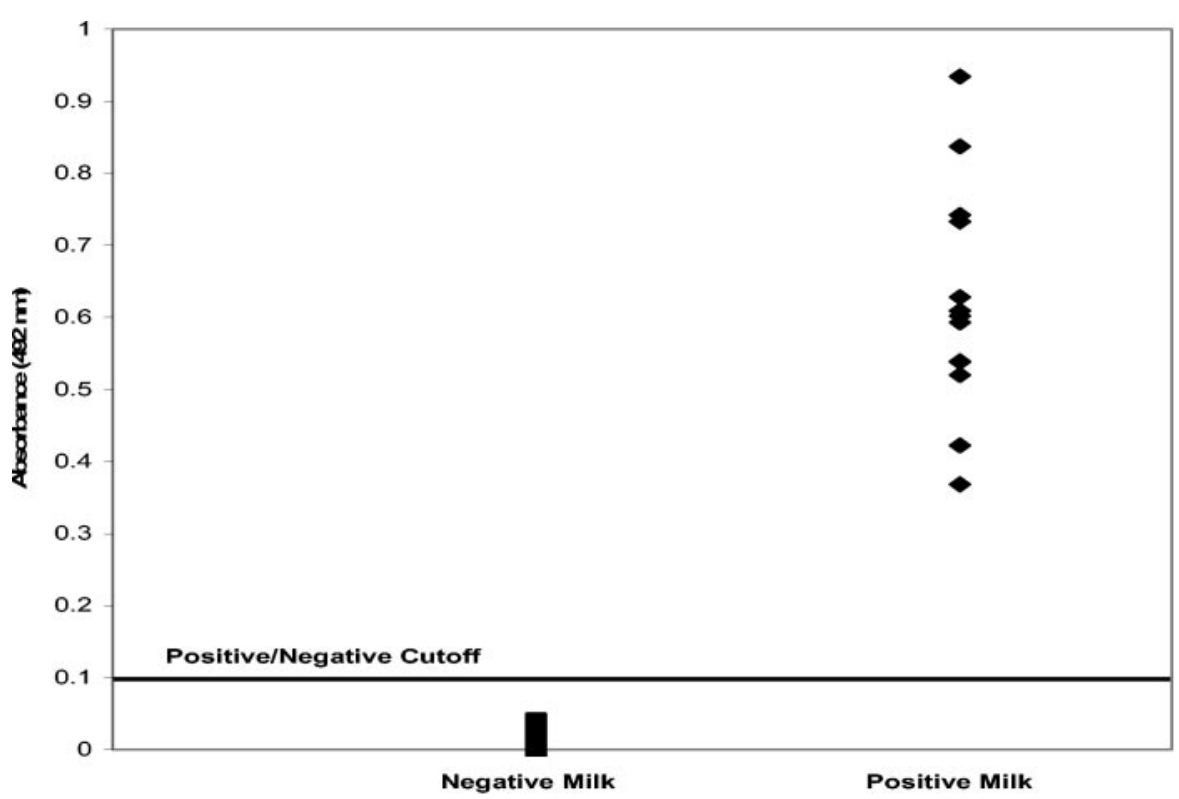

FIG. 2. iELISA results for milk samples. Absorbance data are shown for milk samples from 13 experimentally infected goats ( $)$ and 134 bulk milk samples from uninfected goat herds (ם). Negative milk samples were tested in duplicate at a 1/100 dilution in PBST, and positive milk samples were tested singly. The horizontal line indicates the positive-negative cutoff.

ranged from 0 to 0.0405 . The average positive absorbance was 0.62 (confidence interval, 0.51 to $0.73 ; \alpha=0.01$ ), and the average negative absorbance was 0.0016 (confidence interval, 0.00092 to $0.0023 ; \alpha=0.01$ ). The absorbance values for the negative samples were skewed toward zero, with a median negative absorbance of 0.0. To maximize specificity and maintain sensitivity, we chose a positive-negative cutoff of 0.1 absorbance units. The BCSP iELISA detected all of the individual positive samples $(n=13)$ and did not detect antibodies in any of the negative samples $(n=134)$ (Fig. 2).

Bulk milk simulation. To assess the ability of the assay to detect antibodies in bulk milk, we chose three positive samples (high, medium, and low titers) from the individual milk samples and serially diluted them in $B$. melitensis-negative milk. Each dilution was tested at a 1/10 dilution in PBST (Fig. 3).

TABLE 2. iELISA absorbance and culture data for individual positive milk samples

\begin{tabular}{lccr}
\hline $\begin{array}{c}\text { Sample } \\
\text { no. }\end{array}$ & $\begin{array}{c}\text { Days } \\
\text { postinfection }\end{array}$ & Absorbance $^{a}$ & $\begin{array}{c}\text { CFU of B. melitensis } / \mathrm{ml} \\
\text { of milk }\end{array}$ \\
\hline 13 & 37 & 0.62 & $>10,000$ \\
66 & 28 & 0.59 & $>10,000$ \\
69 & 37 & 0.93 & 120 \\
102 & 37 & 0.73 & 750 \\
$152 \mathrm{~T}$ & 15 & 0.60 & 10 \\
159 & 34 & 0.74 & 10,000 \\
160 & 28 & 0.52 & 5,000 \\
212 & 43 & 0.53 & $>10,000$ \\
214 & 29 & 0.42 & $>10,000$ \\
215 & 43 & 0.84 & 10 \\
220 & 43 & 0.37 & $>10,000$ \\
222 & 50 & 0.61 & 10 \\
251 & 43 & 0.54 & $>10,000$ \\
\hline
\end{tabular}

${ }^{a}$ Absorbance values are for single 1:100 dilutions of milk samples. The mean ( \pm standard deviation) for all absorbance values was $0.62 \pm 0.16$.
The high-titer positive sample was readily detected in milk to a dilution of 1/1,600. The medium- and low-titer positive samples remained positive to $1 / 200$ and $1 / 50$ dilutions, respectively.

\section{DISCUSSION}

The objective of this study was to develop a sensitive and specific diagnostic test for the detection of $B$. melitensis antibodies in goat milk, with the focused application of testing bulk milk samples. BCSP has previously been shown in other investigations to be an efficient antigen for the detection of brucella antibodies in serum (21). Nonspecific cross-reacting antibodies in uninfected animals against brucella lipopolysaccharide (LPS) have been shown for several different pathogens, including Escherichia coli, Yersinia enterocolitica, and Pseudomonas solanacearum $(11,15,17)$. LPS is commonly used as an antigen in most iELISA formats (16). While the presence of LPS in BCSP extract cannot be ruled out (Table 1), the BCSP extract used for our iELISA was based on protein content. The results show that no false-positive results were encountered with uninfected bulk milk samples tested by the BCSP iELISA.

It should be noted that bulk milk samples were used instead of individual milk samples to determine specificity. The primary application for the development of this iELISA was its use for bulk milk samples, and therefore bulk milk was used instead of individual milk samples. Also, because of the ease of collection, a large number of herd samples were obtained from many different areas in the United States, representing $>22,000$ animals. There was no difference noted in the results for uninfected bulk milk samples and those for the individual animals used to make up the pooled negative control. However, the possibility remains that nonspecific antibodies may have been diluted in bulk milk and that the absorbance values for false-positive results therefore fell below the limit of de- 


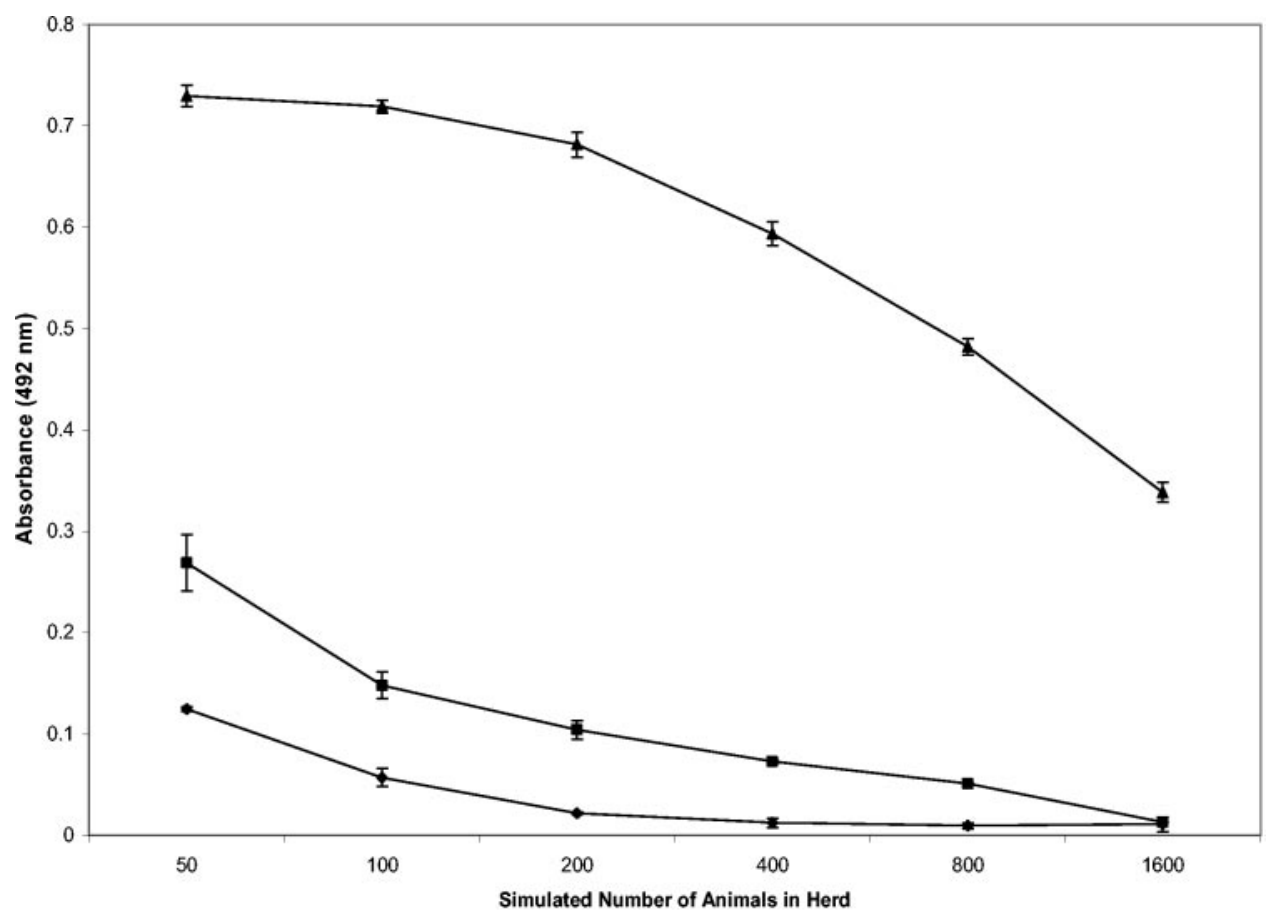

FIG. 3. Bulk milk simulation. iELISA-positive milk samples with high $(\boldsymbol{\Delta})$, medium $(\mathbf{\square})$, or low $(\bullet)$ absorbance values were diluted in negative control milk and then tested in duplicate at a 1/10 dilution in PBST. Bars indicate ranges of absorbance values.

tection. More milk samples from individual animals would be required if this test were to be evaluated for use with individual milk samples.

The iELISA cutoff point was determined by assessing the range between positive and negative samples. Other more empirical methods have been used to set positive-negative cutoff values $(2,6,9)$. Conventional statistical methods for cutoff determination are not applicable given the relatively small number of positive samples available for assay analysis. Ideally, methods such as receiver-operator characteristic analysis would be used to optimize specificity and sensitivity. The range of absorbance values between the highest negative absorbance and the lowest positive absorbance was 0.016 to 0.37 . Any cutoff point determined within this range would give $100 \%$ sensitivity, $100 \%$ specificity, and an area under the curve of 1.0 in a receiver-operator characteristic analysis. The cutoff point of 0.1 was therefore arbitrarily chosen, keeping in mind the need to adjust this value upon testing more positive individual and bulk milk samples. Also, methods such as adding 2 or 3 standard deviations to the mean are not applicable in this case, as the results for the negative samples did not have a normal distribution, which would be required for implementation of this method.

To determine the sensitivity of the assay, we used 13 milk samples from goats that were experimentally infected with B. melitensis strain $16 \mathrm{M}$. Western blotting using BCSP has shown that there is a strong antibody response during infection with $B$. abortus or B. melitensis in cattle and goats, respectively (3; unpublished data). BCSP has also been shown to be effective for detecting $B$. abortus infections in cattle by iELISA (21). Figure 2 shows that goats infected experimentally with B. melitensis produced antibodies against BCSP and that uninfected goats did not.
Because of the scarcity of milk from B. melitensis-infected goats in the field in the United States, samples from experimentally infected goats were used. Note that the immunologic response under experimental conditions may not be equivalent to that seen during natural infections. However, the experimentally infected animals were inoculated with a dose of $10^{7}$ CFU of B. melitensis strain $16 \mathrm{M}$, which has been shown to consistently infect goats and to induce abortions (8). These experimentally infected goats showed high-level immune responses to BCSP prepared from B. abortus strain 1119-3, indicating that there are conserved antigenic elements between species and strains which may be encountered under natural conditions. It is therefore predicted that the antibody response to BCSP during a natural field infection with $B$. melitensis would be similar to those produced experimentally.

Bulk samples containing milk taken from herds that were naturally infected with $B$. melitensis were not available; thus, simulated bulk milk samples containing one positive sample with a high, medium, or low antibody titer were used. For ease of estimation, it was assumed that equal volumes of milk are produced by infected and uninfected animals. Figure 3 shows that one animal with a high antibody titer would be detected in a bulk milk sample from herds with $>1,600$ animals. This number of animals is larger than most herd sizes in the United States. However, if infection produced only a medium or low titer, the ability to detect an infected animal would be limited to a herd size of about 200 animals or less. On the basis of the results shown in Fig. 3, it is recommended that large herds be sampled in groups of 50 animals or less for bulk milk testing.

An unknown factor that was not addressed in this study pertains to the duration of the antibody response. The positive milk samples were collected at necropsy from experimentally 
infected animals from 15 to 50 days postinfection. It is not known how long a positive titer is maintained after infection; therefore, further study would be needed to determine the time course of the antibody response in milk and the frequency of bulk milk testing needed to ensure that brucella-infected animals are detected before infection spreads through the herd.

\section{REFERENCES}

1. Alton, G. G., L. M. Jones, R. D. Angus, and J. M. Verger. 1988. Techniques for the brucellosis laboratory. Institut National de la Recherche, Nouzilly, France.

2. Barajas-Rojas, J. A., H. P. Riemann, and C. E. Franti. 1993. Notes about determining the cut-off value in enzyme-linked immunosorbent assay (ELISA). Prev. Vet. Med. 15:231-233.

3. Belzer, C. A., L. B. Tabatabai, and B. L. Deyoe. 1991. Differentiation by Western blotting of immune responses of cattle vaccinated with Brucella abortus strain 19 or infected experimentally or naturally with virulent Brucella abortus. Vet. Microbiol. 27:79-90.

4. Capasso, L. 1999. Brucellosis at Herculaneum (79 AD). Int. J. Osteoarchaeol. 9:277-288.

5. Corbel, M. J. 1997. Brucellosis: an overview. Emerg. Infect. Dis. 3:213-221.

6. Crowther, J. R. 2001. The ELISA guidebook, p. 189. Humana Press, Totowa, N.J.

7. Dubois, M., K. A. Gilles, T. K. Hamilton, P. A. Rebers, and F. Smith. 1956 Colorimetric method for determination of sugars and related substances. Anal. Chem. 28:350-356.

8. Elzer, P. H., S. D. Hagius, D. S. Davis, V. G. DelVecchio, and F. M. Enright. 2002. Characterization of the caprine model for ruminant brucellosis. Vet. Microbiol. 90:425-431.

9. Greiner, M., D. Pfeiffer, and R. D. Smith. 2000. Principles and practical application of the receiver-operating characteristic analysis for diagnostic tests. Prev. Vet. Med. 45:23-41.

10. Kahler, S. C. 2000. Brucella melitensis infection discovered in cattle for first time, goats also infected. J. Am. Vet. Med. Assoc. 216:648.
11. Lamb, V., L. Jones, G. Schurig, and D. Berman. 1979. Enzyme-linked immunosorbent assay for bovine immunoglobulin subclass-specific response to Brucella abortus lipopolysaccharides. Infect. Immun. 26:240-247.

12. Lowry, O. H., N. J. Rosebrough, A. L. Farr, and R. J. Randall. 1951. Protein measurement with the Folin phenol reagent. J. Biol. Chem. 193:265-275.

13. Mikolon, A. B., I. A. Gardner, S. K. Hietala, J. Hernandez De Anda, E. Chamizo Pestana, S. G. Hennager, and A. J. Edmondson. 1998. Evaluation of North American detection tests for diagnosis of brucellosis in goats. J. Clin. Microbiol. 36:1716-1722.

14. Nielsen, K. 2002. Diagnosis of brucellosis by serology. Vet. Microbiol. 90: 447-459.

15. Nielsen, K., and J. R. Duncan. 1982. Demonstration that nonspecific bovine Brucella abortus agglutinin is EDTA-labile and not calcium-dependent. J. Immunol. 129:366-369.

16. Nielsen, K., and D. Gall. 1994. Advances in the diagnosis of bovine brucellosis: use of enzyme immunoassays. Gen. Eng. Biotechnol. 14:25-39.

17. Nielsen K., K. Stilwell, B. Stemshorn, and R. Duncan. 1981. Ethylenediaminetetraacetic acid (disodium salt)-labile bovine immunoglobulin M Fc binding to Brucella abortus: a cause of nonspecific agglutination. J. Clin. Microbiol. 14:32-38.

18. Rashidi, J. S., D. J. Ortner, B. Frohlich, and B. Jonsdottir. 2001. Brucellosis in early Bronze Age Jordan and Bahrain: an analysis of possible cases of Brucella spondylitis. Am. J. Phys. Anthr. 114:122.

19. Sutra, L., J. P. Caffin, and G. Dubray. 1986. Role of immunoglobulins in the Brucella milk ring test. Vet. Microbiol. 12:359-366.

20. Tabatabai, L. B., B. L. Deyoe, and A. E. Ritchie. 1979. Isolation and characterization of toxic fractions from Brucella abortus. Infect. Immun. 26:668679.

21. Tabatabai, L. B., and B. L. Deyoe. 1984. Specific enzyme-linked immunosorbent assay for detection of bovine antibody to Brucella abortus. J. Clin. Microbiol. 20:209-213.

22. Warren, L. 1959. The thiobarbituric acid assay of sialic acids. J. Biol. Chem. 245:1971-1975.

23. Whiting, R. D., B. M. White, and F. C. Stiles. 1970. An epizootic of Brucella melitensis infection in Texas. J. Am. Vet. Med. Assoc. 157:1860-1863. 